\title{
Some Equivalence Relations and Results over the Commutative Quaternions and Their Matrices
}

\author{
Hidayet Hüda Kösal and Murat Tosun
}

\begin{abstract}
In this paper, we give some equivalence relations and results over the commutative quaternions and their matrices. In this sense, consimilarity, semisimilarity, and consemisimilarity over the commutative quaternion algebra and commutative quaternion matrix algebra are established. Equalities of these equivalence relations are explicitly determined. Also Syvester-s-Conjugate commutative quaternion matrix equations are studied by means of real representation of the commutative quaternion matrices and consimilarity of the two commutative quaternion matrices.
\end{abstract}

\section{Introduction}

In 1843, Hamilton introduced the concept of real quaternions, which is defined by $[1]$

$$
\mathbb{K}=\left\{q=q_{0}+q_{1} i+q_{2} j+q_{3} k: q_{0}, q_{1}, q_{2}, q_{3} \in \mathbb{R} \text { and } i, j, k \notin \mathbb{R}\right\}
$$

where

$$
i^{2}=j^{2}=k^{2}=-1, \quad i j=-j i=k, \quad i k=-k i=-j, \quad j k=-k j=i .
$$

Key Words: Commutative quaternion, equivalence relation, coneigenvalue, Slyvester conjugate matrix equation.

2010 Mathematics Subject Classification: Primary 15B33; Secondary 15A24.

Received: 20.11 .2016

Revised: 15.12 .2016

Accepted: 21.12.2016 
The real quaternion algebra plays an important role in quantum physics, kinematic, differential geometry, game development, image processing and signal processing, etc. Real quaternions are a naturel extension of the complex numbers which are also the extension of the real numbers. The multiplication of real quaternions is non-commutative. Thus, all results about complex numbers cannot be generalized in real quaternions. There are a lot of works associated with real quaternions. For instance, Tian defined two types of universal factorization equalities for real quaternions and gave solutions of $a x-x b=c$ in $\mathbb{K},[2]$. As well as the similarity and consimilarity of elements of the real quaternion, octanion, and sedenion algebras, Tian considered the similarity and consimilarity of general real Cayley-Dickson algebras in [3]. Also, the author studied the solutions of two fundamental equations, $a x=x b$ and $a x=\bar{x} b$, by means of similarity and consimilarity relations. In [4], Tian defined semisimilarity and consemisimilarity of real quaternions and investigated the general solutions of systems $x a y=b, y b x=a$, and $\bar{x} a y=b, \bar{y} b x=a$ in $\mathbb{K}$. One of the applications of real quaternions is also the quaternion matrix theory. In [5], Baker investigated the right eigenvalues of the quaternion matrices using the topological approach. Besides, Huang and So studied on the left eigenvalues of real quaternion matrices [6]. Huang discussed the consimilarity of the quaternion matrices and obtained their Jordan canonical form, using the consimilarity [7]. Jiang and Wei studied the Kalman-Yakubovich-Conjugate matrix equation , $X-A \widetilde{X} B=C$, in $\mathbb{K}$ (where $\widetilde{X}=-j X j$ ) via the real representation of real quaternion matrices [8]. Moreover, Jiang and Ling studied the problem of the solution of the Sylvester-Conjugate real quaternion matrix equation , $A \widetilde{X}-X B=C$, by means of real representation of the real quaternions matrix $[9]$.

After the introduction of real quaternions, the set of commutative quaternions was first introduced by Segre [10]. This number system is sometimes called the system of reduced bi-quaternion. The set of commutative quaternions is four-dimensional like the set of real quaternions. However this set contains zero-divisor elements. Commutative quaternions are extensively studied and applied to several problems in various areas. Catoni et al. studied the functions of commutative quaternion variable and obtained generalized Cauchy-Riemann conditions [11]. In [12], the authors introduced digital signal and image processing, using commutative quaternions. Also, they discussed the efficient algorithms of the discrete commutative quaternion Fourier transform, convolution, correlation, and phase-only correlation. In [13], the authors developed the algorithms for calculating the eigenvalues-eigenvectors and the singular value decomposition of commutative quaternion matrices. Moreover, they represented the color images in the digital media by commutative quaternion matrices and applied the techniques, such as separation, 
compression, image enhancement and denosing, to the color images by using these matrices. In [14], the authors investigated two types of multistate Hopfield neural networks based on commutative quaternions. In [15], the authors defined commutative quaternion canonical transform which is the generalization of commutative quaternion Fourier transform. Also, Kosal and Tosun investigated some algebraic properties of commutative quaternion matrices by means of complex representation of commutative quatrnion matrices [16]. In [17], Kosal et al. constructed, by means of real representation of a real matrix, some explicit expression of the solutions of the matrix equations, $X-A \overline{\bar{X}} B=C, X-A \overline{\widetilde{X}} B=C$, and $X-A \widetilde{\bar{X}} B=C$, which, for convenience, are called the Kalman-Yakubovich-Conjugate commutative quaternion matrix equations.

This article is organized as follows. In Section 2, after we give algebraic properties of commutative quaternions, consimilarity, semisimilarity and consemisimilarity of commutative quaternions are defined. Also, equalities of these equivalence relations are studied. In Section 3, we define consimilarity, semisimilarity, and consemisimilarity of commutative quaternion matrices and obtain equalities of these equivalence relations. Lastly, we introduce Syvester-s-Conjugate matrix equations, $A\left({ }^{s} \bar{X}\right)-X B=C,(s=1,2,3)$ via real representation of commutative quaternion matrices and consimilarity of the two commutative quaternion matrices. Throughout this paper, the following notations are used. Let $\mathbb{R}, \mathbb{K}$, and $\mathbb{H}$ denote the real numbers field, the real quaternion skew field, and the commutative quaternion ring, respectively. $\mathbb{R}^{m \times n}\left(\mathbb{K}^{m \times n}\right.$ or $\left.\mathbb{H}^{m \times n}\right)$ denotes the set of all matrices on $\mathbb{R}(\mathbb{K}$ or $\mathbb{H})$.

\section{Equivalence Relations and Results over the Commu- tative Quaternions}

The set of commutative quaternions is expressed by

$$
\mathbb{H}=\left\{a=a_{0}+a_{1} i+a_{2} j+a_{3} k: a_{0}, a_{1}, a_{2}, a_{3} \in \mathbb{R} \text { and } i, j, k \notin \mathbb{R}\right\},
$$

where

$$
i^{2}=k^{2}=-1, j^{2}=1, i j=j i=k, j k=k j=i, k i=i k=-j .
$$

It is clear that multiplication in $\mathbb{H}$ is commutative. Summation of the commutative quaternions $a=a_{0}+a_{1} i+a_{2} j+a_{3} k, \quad b=b_{0}+b_{1} i+b_{2} j+b_{3} k \in \mathbb{H}$ is defined as $a+b=\left(a_{0}+b_{0}\right)+\left(a_{1}+b_{1}\right) i+\left(a_{2}+b_{2}\right) j+\left(a_{3}+b_{3}\right) k$. Scalar multiplication of a commutative quaternion $a \in \mathbb{H}$ with a scalar $\lambda \in \mathbb{R}$ is defined as $\lambda a=\lambda\left(a_{0}+a_{1} i+a_{2} j+a_{3} k\right)=\lambda a_{0}+\lambda a_{1} i+\lambda a_{2} j+\lambda a_{3} k$. In addition, quaternionic multiplication of two commutative quaternions $a, b \in \mathbb{H}$ 
is defined

$$
\begin{aligned}
& a b=\left(a_{0} b_{0}-a_{1} b_{1}+a_{2} b_{2}-a_{3} b_{3}\right)+\left(a_{1} b_{0}+a_{0} b_{1}+a_{3} b_{2}+a_{2} b_{3}\right) i \\
& \quad+\left(a_{0} b_{2}+a_{2} b_{0}-a_{1} b_{3}-a_{3} b_{1}\right) j+\left(a_{3} b_{0}+a_{0} b_{3}+a_{1} b_{2}+a_{2} b_{1}\right) k .
\end{aligned}
$$

There are three types of conjugates of $a \in \mathbb{H}$. They are ${ }^{1} \bar{a}=a_{0}-a_{1} i+a_{2} j-$ $a_{3} k,{ }^{2} \bar{a}=a_{0}+a_{1} i-a_{2} j-a_{3} k$, and ${ }^{3} \bar{a}=a_{0}-a_{1} i-a_{2} j+a_{3} k$. Also, the norm of $a \in \mathbb{H}$ is defined as

$$
\|a\|=\sqrt[4]{a\left({ }^{\bar{a}}\right)\left({ }^{2} \bar{a}\right)\left({ }^{3} \bar{a}\right)}=\sqrt[4]{\left[\left(a_{0}+a_{2}\right)^{2}+\left(a_{1}+a_{3}\right)^{2}\right]\left[\left(a_{0}-a_{2}\right)^{2}+\left(a_{1}-a_{3}\right)^{2}\right]} .
$$

In case of

$$
a_{0}+a_{2}=0, a_{1}+a_{3}=0 \text { or } a_{0}-a_{2}=0, a_{1}-a_{3}=0
$$

norm of $a$ is equal to zero. The planes of equations (3) are called planes of the zero divisors or characteristic planes [11].

If $a \in \mathbb{H}$ and $\|a\| \neq 0$ then $a$ has multiplicative inverse. Multiplicative inverse of $a$ is equal to $a^{-1}=\frac{\left({ }^{1} \bar{a}\right)\left({ }^{2} \bar{a}\right)\left({ }^{3} \bar{a}\right)}{\|a\|^{4}}[11]$.

It is nearby to identify a commutative quaternion $a \in \mathbb{H}$ with a real vector $\boldsymbol{a} \in \mathbb{R}^{4}$. We may define any commutative quaternion as

$$
a=a_{0}+a_{1} i+a_{2} j+a_{3} k \cong \boldsymbol{a}=\left(\begin{array}{c}
a_{0} \\
a_{1} \\
a_{2} \\
a_{3}
\end{array}\right) .
$$

Then multiplication of $a$ and $b$ can be shown, with the help of ordinary matrix multiplication,

$$
a b=b a \cong\left(\begin{array}{cccc}
a_{0} & -a_{1} & a_{2} & -a_{3} \\
a_{1} & a_{0} & a_{3} & a_{2} \\
a_{2} & -a_{3} & a_{0} & -a_{1} \\
a_{3} & a_{2} & a_{1} & a_{0}
\end{array}\right)\left(\begin{array}{c}
b_{0} \\
b_{1} \\
b_{2} \\
b_{3}
\end{array}\right)=\varphi(a) \boldsymbol{b},
$$

where $\varphi(a)$ is sometimes called fundamental matrix of $a$ [11].

Theorem 1. ([12]) Let $a, b \in \mathbb{H}$ and $\lambda \in \mathbb{R}$. Then the following identities hold:

1. $\varphi(a b)=\varphi(a) \varphi(b)$,

2. $\varphi(\varphi(a) \boldsymbol{b})=\varphi(a) \varphi(b)$,

3. $a=b \Leftrightarrow \varphi(a)=\varphi(b)$,

4. $\varphi(a+b)=\varphi(a)+\varphi(b)$,

5. $\varphi(\lambda a)=\lambda \varphi(a)$,

6. $\operatorname{trace}(\varphi(a))=a+{ }^{1} \bar{a}+{ }^{2} \bar{a}+{ }^{3} \bar{a}=4 a_{0}$,

7. $\|a\|^{4}=|\operatorname{det}(\varphi(a))|$. 
Definition 1. Two commutative quaternions $a$ and $b$ said to be consimilar according to $s^{t h}(s=1,2,3)$ conjugate if there exists a commutative quaternion $p,\|p\| \neq 0$ such that ${ }^{s} \bar{p} a p^{-1}=b$; this is written as $a \stackrel{c_{s}}{\sim} b$. Consimilarity relation $\stackrel{c}{\sim}$ is an equivalence relation on the commutative quaternions.

Theorem 2. Let $a, b \in \mathbb{H}$ and a is consimilar to $b$ according to $s^{\text {th }}(s=1,2,3)$ conjugate. Then norm of a is equal to norm of $b$.

Proof. If $a$ and $b$ are consimilar according to $s^{t h}(s=1,2,3)$ conjugate, then there exists $p$ such as

$$
{ }^{s} \bar{p} a p^{-1}=b .
$$

In last equation, we can calculate the norm both sides. Then we have

$$
\left\|^{s} \bar{p}\right\|\|a\|\left\|p^{-1}\right\|=\|b\|
$$

Since $\left\|^{s} \bar{p}\right\|=\|p\|$ and $\left\|p^{-1}\right\|=\|p\|^{-1}$, we get $\|a\|=\|b\|$.

Definition 2. Two commutative quaternions $a$ and $b$ are said to be semisimilar if there exist a commutative quaternions $x$ and $y$ that satisfying the equation

$$
x a y=b \text { and } y b x=a,
$$

this is written as $a \approx b$. Semisimilarity relation $\approx$ is an equivalence relation on $\mathbb{H}$.

Theorem 3. Let $a, b \in \mathbb{H}$ are semisimilar and $\|a\| \neq 0,\|b\| \neq 0$. Then $\|a\|=\|b\|$ and $a^{2}=b^{2}$.

Proof. Let $a \approx b$ and $\|a\| \neq 0,\|b\| \neq 0$. Then there exist $x$ and $y$ such that $x a y=b, y b x=a$. Thus we get

$$
\frac{\|a\|}{\|b\|}=\frac{\|b\|}{\|a\|}
$$

that is $\|a\|=\|b\|$.

If the right side of first equation in (5) multiplied by $y^{-1} a^{-1}$, then we obtain $x=b y^{-1} a^{-1}$. Substitution $x=b y^{-1} a^{-1}$ into the second equation in (5) and simplifying gives us $y b^{2} y^{-1}=a^{2}$ that is $a^{2}=b^{2}$.

Theorem 4. Let $x, y, a, b \in \mathbb{H},\|a\| \neq 0,\|b\| \neq 0$ and $a \approx b$. Then there exist $x, y \in \mathbb{H}$ satisfying

$$
x a y=b, y b x=a
$$

and

$$
x=b q_{2}^{-1} a^{-1}, y=q_{2}
$$


or

$$
x=q_{1}, \quad y=a q_{1}^{-1} b^{-1}
$$

where $q_{1}, q_{2} \in \mathbb{H}$ are arbitrary and $\left\|q_{1}\right\|,\left\|q_{2}\right\| \neq 0$.

Proof. If $\|a\| \neq 0$ and $\|b\| \neq 0$, then the norms of $x$ and $y$ satisfying (6) are nonzero. If the right side of first equation in (6) multiplied by $y^{-1} a^{-1}$, then we obtain $x=b y^{-1} a^{-1}$. Substitution $x=b y^{-1} a^{-1}$ into the second equation in (6) gives us

$$
y b^{2}=y a^{2} .
$$

Similarly, getting solution for $y$ from the second equation in (6) and subsitituting it into the first equation in (6) gives

$$
x b^{2}=x a^{2} .
$$

Since $a \approx b$, we get $a^{2}=b^{2}$. In the present case, $y$ that satisfies (9) is arbitrary. Substituting this $y$ into the first equation in (6) gives (7). Solving for $x$ in (10) and substituting this $x$ into the second equation in (6) gives (8).

Definition 3. Two commutative quaternions $a$ and $b$ are consemisimilar according to $s^{\text {th }}$ conjugate if there exist a commutative quaternions $x$ and $y$ satisfying

$$
\left({ }^{s} \bar{x}\right) a y=b, \quad\left({ }^{s} \bar{y}\right) b x=a, \quad s=1,2,3,
$$

this is written as $a \stackrel{c_{s}}{\approx} b$. Consemisimilarity relation $\stackrel{c_{s}}{\approx}$ is an equivalence relation on $\mathbb{H}$.

Theorem 5. Let $\|a\| \neq 0,\|b\| \neq 0$ and $a, b \in \mathbb{H}$ are consemisimilar according to $s^{\text {th }}(s=1,2,3)$ conjugate. Then $\|a\|=\|b\|$ and $\left({ }^{s} \bar{a}\right) a=\left({ }^{s} \bar{b}\right) b$.

The proof of Theorem 8 may be proved along the same way as the proof of Theorem 5 .

Theorem 6. Let $x, y, a, b \in \mathbb{H},\|a\| \neq 0,\|b\| \neq 0$ and $a \stackrel{c_{s}}{\approx} b$. Then there exist $x, y \in \mathbb{H}$ satisfying

$$
\left({ }^{s} \bar{x}\right) a y=b,\left({ }^{s} \bar{y}\right) b x=a
$$

and

$$
x=\left({ }^{s} \bar{b}\right)\left({ }^{s} \overline{q_{2}}\right)^{-1}\left({ }^{s} \bar{a}\right)^{-1}, \quad y=q_{2}
$$

or

$$
x=q_{1}, \quad y=\left({ }^{s} \bar{a}\right)\left({ }^{s} \overline{q_{1}}\right)^{-1}\left({ }^{s} \bar{b}\right)^{-1}
$$

where $q_{1}, q_{2} \in \mathbb{H}$ are arbitrary and $\left\|q_{1}\right\|,\left\|q_{2}\right\| \neq 0$. 
Proof. If $\|a\| \neq 0$ and $\|b\| \neq 0$, then the norms of $x$ and $y$ satisfying (11) are nonzero. If the right side of first equation in (11) multiplied by $y^{-1} a^{-1}$, then we get $x=\left({ }^{s} \bar{b}\right)\left({ }^{s} \bar{y}\right)^{-1}\left({ }^{s} \bar{a}\right)^{-1}$. Substitution $x=\left({ }^{s} \bar{b}\right)\left({ }^{s} \bar{y}\right)^{-1}\left({ }^{s} \bar{a}\right)^{-1}$ into the second equation in (11) gives us

$$
y\left({ }^{s} \bar{b}\right) b=y\left({ }^{s} \bar{a}\right) a .
$$

In a similar way, getting solution for $y$ from the second equation in (11) and subsitituting it into the first equation in (11) gives

$$
x\left({ }^{s} \bar{b}\right) b=x\left({ }^{s} \bar{a}\right) a .
$$

Since $a \stackrel{c_{s}}{\approx} b$, we get $\left({ }^{s} \bar{a}\right) a=\left({ }^{s} \bar{b}\right) b$. In the circumstances, $y$ that satisfies (14) is arbitrary. Substituting this $y$ into the first equation in (11) gives (12). Solving for $x$ in (15) and substituting this $x$ into the second equation in (11) gives (13).

\section{Equivalence Relations and Results over the Commu- tative Quaternions Matrices}

The set of $m \times n$ matrices with commutative quaternion entries, which is denoted by $\mathbb{H}^{m \times n}$, with usual matrix summation and multiplication is a ring with unity. There exist three kinds of conjugate of $A=\left(a_{i j}\right) \in \mathbb{H}^{m \times n}$ and they are ${ }^{1} \bar{A}=\left({ }^{1} \overline{a_{i j}}\right) \in \mathbb{H}^{m \times n},{ }^{2} \bar{A}=\left({ }^{2} \overline{a_{i j}}\right) \in \mathbb{H}^{m \times n}$ and ${ }^{3} \bar{A}=\left({ }^{3} \overline{a_{i j}}\right) \in \mathbb{H}^{m \times n}$. A matrix $A^{T} \in \mathbb{H}^{n \times m}$ is transpose of $A \in \mathbb{H}^{m \times n}$. Also $A^{* s}=\left({ }^{s} \bar{A}\right)^{T} \in \mathbb{H}^{n \times m}$ is called conjugate transpose according to $s^{t h}(s=1,2,3)$ conjugate of $A \in$ $\mathbb{H}^{m \times n},[16]$.

Theorem 7. ([17]) Let $A$ and $B$ be matrices of appropriate sizes. Then followings are satisfied:

1. $\left({ }^{s} \bar{A}\right)^{T}=s \overline{\left(A^{T}\right)}$,

2. $(A B)^{*_{s}}=B^{*_{s}} A^{* s}$,

3. $(A B)^{T}=B^{T} A^{T}$,

4. ${ }^{s} \overline{(A B)}=\left({ }^{s} \bar{A}\right)\left({ }^{s} \bar{B}\right)$,

5. If $A^{-1}$ and $B^{-1}$ exist then $(A B)^{-1}=B^{-1} A^{-1}$,

6. If $A^{-1}$ exists then $\left(A^{* s}\right)^{-1}=\left(A^{-1}\right)^{*_{s}}$,

7. $(s \bar{A})^{-1}=s \overline{\left(A^{-1}\right)}$.

Definition 4. Two commutative quaternion matrices $A, B \in \mathbb{H}^{n \times n}$ said to be consimilar according to $s^{t h}(s=1,2,3)$ conjugate if there exists a commutative quaternion $P \in \mathbb{H}^{n \times n}$ such that ${ }^{s} \bar{P} A P^{-1}=B$; this is written as $A \stackrel{c_{s}}{\sim} B$. 
Definition 5. ([17]) Let $A \in \mathbb{H}^{n \times n}, \lambda \in \mathbb{H}$. If there exists $0 \neq x \in \mathbb{H}^{n \times 1}$ such that

$$
A x=x \lambda
$$

then $\lambda$ is said to be eigenvalues of $A$ and $x$ is said to be a eigenvector of $A$ corresponding to the eigenvalue $\lambda$. The set of eigenvalues is defined as

$$
\xi(A)=\left\{\lambda \in \mathbb{H}: A x=\lambda x, \text { for some } 0 \neq x \in \mathbb{H}^{n \times 1}\right\} .
$$

Definition 6. Let $A \in \mathbb{H}^{n \times n}, \lambda \in \mathbb{H}$. If there exists $0 \neq x \in \mathbb{H}^{n \times 1}$ such that

$$
A\left({ }^{s} \bar{x}\right)=x \lambda
$$

then $\lambda$ is said to be coneigenvalues according to $s^{\text {th }}$ conjugate of $A$ and $x$ is said to be a coneigenvector of $A$ corresponding to the coneigenvalue $\lambda$. The set of coneigenvalues according to $s^{\text {th }}$ conjugate is denoted as

$$
\bar{\sigma}=\left\{\lambda \in \mathbb{H}: A\left({ }^{s} \bar{x}\right)=x \lambda \text {, for some } 0 \neq x \in \mathbb{H}^{n \times 1}\right\} .
$$

Theorem 8. Let $A, B \in \mathbb{H}^{n \times n}$. If $A \stackrel{c_{s}}{\sim} B$, then $A$ and $B$ have the same coneigenvalues according to $s^{\text {th }}$ conjugate.

Proof. Let $A \stackrel{c_{s}}{\sim} B$, then, there exists a regular matrix $P \in \mathbb{H}^{n \times n}$ such that ${ }^{s} \bar{P} A P^{-1}=B$. Let $\lambda \in \mathbb{H}$ be a coneigenvalue according to $s^{t h}$ conjugate for the matrix $A$, then we find the matrix $x \in \mathbb{H}^{n \times 1}$ such that $A\left({ }^{s} \bar{x}\right)=x \lambda, 0 \neq$ $x \in \mathbb{H}^{n \times 1}$. Let $y=P\left({ }^{s} \bar{x}\right)$. Then

$$
B y={ }^{s} \bar{P} A P^{-1} y={ }^{s} \bar{P} A P^{-1} P\left({ }^{s} \bar{x}\right)={ }^{s} \bar{P} A\left({ }^{s} \bar{x}\right)={ }^{s} \bar{P} x \lambda={ }^{s} \bar{y} \lambda .
$$

Thus, $A$ and $B$ have the same coneigenvalues according to $s^{\text {th }}$ conjugate.

Theorem 9. If $A \in \mathbb{H}^{n \times n}$, then $\lambda$ is coneigenvalue according to $s^{\text {th }}$ conjugate of $A$ if and only if for any $\beta \in \mathbb{H},(0 \neq\|\beta\|),\left({ }^{s} \bar{\beta}\right) \lambda \beta^{-1}$ is a coneigenvalue according to $s^{\text {th }}$ conjugate of $A$.

Proof. From $A\left({ }^{s} \bar{x}\right)=x \lambda$, we get $A\left({ }^{s} \bar{x} \beta^{-1}\right)=x\left({ }^{s} \bar{\beta}\right)^{-1}\left({ }^{s} \bar{\beta}\right) \lambda \beta^{-1}$. Thus $\lambda$ is coneigenvalue according to $s^{t h}$ conjugate of $A$ if and only if any $\beta \in \mathbb{H},(0 \neq$ $\|\beta\|),\left({ }^{s} \bar{\beta}\right) \lambda \beta^{-1}$ is a coneigenvalue according to $s^{\text {th }}$ conjugate of $A$.

Definition 7. Two commutative quaternion matrices $A \in \mathbb{H}^{n \times n}$ and $B \in$ $\mathbb{H}^{n \times n}$ are said to be semisimilar if there exist a commutative quaternion matrices $X$ and $Y$ that satisfying the equation

$$
Y A X=B, \quad X B Y=A .
$$

This is written as $A \approx B$. 
Definition 8. Two commutative quaternion matrices $A \in \mathbb{H}^{n \times n}$ and $B \in$ $\mathbb{H}^{n \times n}$ are said to be consemisimilar according to $s^{t h}$ conjugate if there exist a commutative quaternion matrices $X$ and $Y$ that satisfying the equation

$$
\left({ }^{s} \bar{Y}\right) A X=B, \quad\left({ }^{s} \bar{X}\right) B Y=A .
$$

This is written as $A \stackrel{c s}{\approx} B$.

Theorem 10. Let $A, B, X, Y \in \mathbb{H}^{n \times n}$ and $A$ is semisimilar to $B$. Then the following hold:

1. $A^{2 k} X=X B^{2 k}, \quad B^{2 k} X=X A^{2 k}, \quad k=1,2,3, \ldots$,

2. $(X Y)^{k} A(X Y)^{k}=A, \quad k=1,2,3, \ldots$,

Proof. 1. Since $Y A X=B$ and $X B Y=A$,

$$
A=X B Y=(X Y) A(X Y) \text { and } B=Y A X=(Y X) B(Y X) .
$$

Then

$$
A^{2} X=(X B Y)(X B Y) X=X B^{2} \text { and } B^{2} Y=(Y A X)(Y A X) Y=Y A^{2},
$$

by induction we get

$$
A^{2 k} X=X B^{2 k}, B^{2 k} X=X A^{2 k}, k=1,2,3, \ldots
$$

2. From the proof of part 1 , It is easily follows by induction that

$$
\begin{aligned}
& A=(X Y) A(X Y)=(X Y) X B Y(X Y)=(X Y) X(Y A X) Y(X Y) \\
& =(X Y) X(Y(X B Y) X) Y(X Y)=(X Y) X(Y(X(Y A X) Y) X) Y(X Y) \\
& \quad \cdots \\
& =(X Y)^{k} A(X Y)^{k}, k=1,2,3, \ldots
\end{aligned}
$$

Theorem 11. Let $A, B, X, Y \in \mathbb{H}^{n \times n}, A \stackrel{c}{\approx} B$. Then the following hold:

1. $\left(\left({ }^{s} \bar{X}\right)\left({ }^{s} \bar{Y}\right)\right)^{k} A(X Y)^{k}=A,\left(\left({ }^{s} \bar{Y}\right)\left({ }^{s} \bar{X}\right)\right)^{k} B(Y X)^{k}=B, k=1,2,3, \ldots$,

2. $\left(A\left({ }^{s} \bar{A}\right)\right)^{k}\left({ }^{s} \bar{X}\right)=\left({ }^{s} \bar{X}\right)\left(B\left({ }^{s} \bar{B}\right)\right)^{k},\left({ }^{s} \bar{Y}\right)\left(A\left({ }^{s} \bar{A}\right)\right)^{k}=\left(B\left({ }^{s} \bar{B}\right)\right)^{k}\left({ }^{s} \bar{Y}\right)$, $k=1,2,3, \ldots$

Proof. 1. Since

$$
\left({ }^{s} \bar{Y}\right) A X=B, \quad\left({ }^{s} \bar{X}\right) B Y=A
$$

We have

$$
A=\left({ }^{s} \bar{X}\right) B Y=\left(\left({ }^{s} \bar{X}\right)\left({ }^{s} \bar{Y}\right)\right) A(X Y)=\left(\left({ }^{s} \bar{X}\right)\left({ }^{s} \bar{Y}\right)\right)\left({ }^{s} \bar{X}\right) B Y(X Y)
$$




$$
\begin{aligned}
& =\left(\left({ }^{s} \bar{X}\right)\left({ }^{s} \bar{Y}\right)\right)\left({ }^{s} \bar{X}\right)\left(\left({ }^{s} \bar{Y}\right) A X\right) Y(X Y) \\
& =\left(\left({ }^{s} \bar{X}\right)\left({ }^{s} \bar{Y}\right)\right)\left({ }^{s} \bar{X}\right)\left(\left({ }^{s} \bar{Y}\right)\left(\left({ }^{s} \bar{X}\right) B Y\right) X\right) Y(X Y) \\
& \cdots \\
& =\left(\left({ }^{s} \bar{X}\right)\left({ }^{s} \bar{Y}\right)\right)^{k} A\left(\left({ }^{s} \bar{X}\right)\left({ }^{s} \bar{Y}\right)\right)^{k}, \quad k=1,2,3, \ldots
\end{aligned}
$$

Symmetry yields the $\left(\left({ }^{s} \bar{Y}\right)\left({ }^{s} \bar{X}\right)\right)^{k} B(Y X)^{k}=B$ for $k=1,2,3, \ldots$

2. From the proof of part $1, B=\left({ }^{s} \bar{Y}\right)\left({ }^{s} \bar{X}\right) B Y X$. Then we have

$$
\begin{gathered}
A\left({ }^{s} \bar{A}\right)\left({ }^{s} \bar{X}\right)=\left({ }^{s} \bar{X}\right) B Y X\left({ }^{s} \bar{B}\right)\left({ }^{s} \bar{Y}\right)\left({ }^{s} \bar{X}\right)=\left({ }^{\prime} \bar{X}\right) B\left(Y X\left({ }^{s} \bar{B}\right)\left({ }^{s} \bar{Y}\right)\left({ }^{s} \bar{X}\right)\right) \\
=\left({ }^{s} \bar{X}\right) B\left({ }^{s} \bar{B}\right)
\end{gathered}
$$

which may be applied repeatedly to obtain $\left(A\left({ }^{s} \bar{A}\right)\right)^{k}\left({ }^{s} \bar{X}\right)=\left({ }^{s} \bar{X}\right)\left(B\left({ }^{s} \bar{B}\right)\right)^{k}$ for $k=1,2,3, \ldots$ Symmetry yields the $\left({ }^{s} \bar{Y}\right)\left(A\left({ }^{s} \bar{A}\right)\right)^{k}=\left(B\left({ }^{s} \bar{B}\right)\right)^{k}\left({ }^{s} \bar{Y}\right)$ for $k=1,2,3, \ldots$

\subsection{Real Representations of Commutative Quaternion Matrices}

Let $A=A_{0}+A_{1} i+A_{2} j+A_{3} k \in \mathbb{H}^{m \times n}$ where $A_{0}, A_{1}, A_{2}, A_{3} \in \mathbb{R}^{m \times n}$. We will define the linear transformations $\phi_{A}(X)=A\left({ }^{1} \bar{X}\right), \mu_{A}(X)=A\left({ }^{2} \bar{X}\right)$ and $\eta_{A}(X)=A\left({ }^{3} \bar{X}\right)$.

Matrices of these linear transformations according to basis $\{1, i, j, k\}$ are

$$
\begin{aligned}
\phi_{A}=\left(\begin{array}{cccc}
A_{0} & A_{1} & A_{2} & A_{3} \\
A_{1} & -A_{0} & A_{3} & -A_{2} \\
A_{2} & A_{3} & A_{0} & A_{1} \\
A_{3} & -A_{2} & A_{1} & -A_{0}
\end{array}\right) \in \mathbb{R}^{4 m \times 4 n}, \mu_{A}=\left(\begin{array}{cccc}
A_{0} & -A_{1} & -A_{2} & A_{3} \\
A_{1} & A_{0} & -A_{3} & -A_{2} \\
A_{2} & -A_{3} & -A_{0} & A_{1} \\
A_{3} & A_{2} & -A_{1} & -A_{0}
\end{array}\right) \in \mathbb{R}^{4 m \times 4 n,} \\
\eta_{A}=\left(\begin{array}{cccc}
A_{0} & A_{1} & -A_{2} & -A_{3} \\
A_{1} & -A_{0} & -A_{3} & A_{2} \\
A_{2} & A_{3} & -A_{0} & -A_{1} \\
A_{3} & -A_{2} & -A_{1} & A_{0}
\end{array}\right) \in \mathbb{R}^{4 m \times 4 n}
\end{aligned}
$$

respectively [17].

Theorem 12. ([17]) Let $A \in \mathbb{H}^{m \times n}$. The following identities are satisfied:

1. If $A \in \mathbb{H}^{m \times n}$, then

$$
\begin{aligned}
& \left({ }^{1} P_{m}\right)^{-1} \phi_{A}\left({ }^{1} P_{n}\right)=\phi_{\left({ }^{1} \bar{A}\right)}, Q_{m}^{-1} \phi_{A} Q_{n}=-\phi_{A}, R_{m}^{-1} \phi_{A} R_{n}=\phi_{A}, S_{m}^{-1} \phi_{A} S_{n}=-\phi_{A}, \\
& \left({ }^{2} P_{m}\right)^{-1} \mu_{A}\left({ }^{2} P_{m}\right)=\phi_{(2 \bar{A})}, Q_{m}^{-1} \mu_{A} Q_{n}=\phi_{A}, R_{m}^{-1} \mu_{A} R_{n}=-\phi_{A}, S_{m}^{-1} \mu_{A} S_{n}=-\phi_{A}
\end{aligned}
$$


and

$$
\left({ }^{3} P_{m}\right)^{-1} \eta_{A}\left({ }^{3} P_{m}\right)=\eta_{(3 \bar{A})}, Q_{m}^{-1} \eta_{A} Q_{n}=-\eta_{A}, R_{m}^{-1} \eta_{A} R_{n}=-\eta_{A}, \quad S_{m}^{-1} \eta_{A} S_{n}=\eta_{A}
$$

where

$$
\begin{aligned}
{ }^{1} P_{t} & =\left(\begin{array}{cccc}
I_{t} & 0 & 0 & 0 \\
0 & -I_{t} & 0 & 0 \\
0 & 0 & I_{t} & 0 \\
0 & 0 & 0 & -I_{t}
\end{array}\right),{ }^{2} P_{t}=\left(\begin{array}{cccc}
I_{t} & 0 & 0 & 0 \\
0 & I_{t} & 0 & 0 \\
0 & 0 & -I_{t} & 0 \\
0 & 0 & 0 & -I_{t}
\end{array}\right),{ }^{3} P_{t}=\left(\begin{array}{cccc}
I_{t} & 0 & 0 & 0 \\
0 & -I_{t} & 0 & 0 \\
0 & 0 & -I_{t} & 0 \\
0 & 0 & 0 & I_{t}
\end{array}\right) \\
Q_{t} & =\left(\begin{array}{cccc}
0 & -I_{t} & 0 & 0 \\
I_{t} & 0 & 0 & 0 \\
0 & 0 & 0 & -I_{t} \\
0 & 0 & I_{t} & 0
\end{array}\right), R_{t}=\left(\begin{array}{cccc}
0 & 0 & I_{t} & 0 \\
0 & 0 & 0 & I_{t} \\
I_{t} & 0 & 0 & 0 \\
0 & I_{t} & 0 & 0
\end{array}\right), S_{t}=\left(\begin{array}{cccc}
0 & 0 & 0 & -I_{t} \\
0 & 0 & I_{t} & 0 \\
0 & -I_{t} & 0 & 0 \\
I_{t} & 0 & 0 & 0
\end{array}\right) .
\end{aligned}
$$

2. If $A, B \in \mathbb{H}^{m \times n}$ then $\phi_{A+B}=\phi_{A}+\phi_{B}, \mu_{A+B}=\mu_{A}+\mu_{B}$ and $\eta_{A+B}=$ $\eta_{A}+\eta_{B}$.

3. If $A \in \mathbb{H}^{m \times n}, B \in \mathbb{H}^{n \times l}$, then

$$
\phi_{A B}=\phi_{A}\left({ }^{1} P_{n}\right) \phi_{B}=\phi_{A} \phi_{\left({ }^{1} \bar{B}\right)}\left({ }^{1} P_{l}\right), \mu_{A B}=\mu_{A}\left({ }^{2} P_{n}\right) \mu_{B}=\mu_{A} \mu_{(2 \bar{B})}\left({ }^{2} P_{l}\right)
$$

and

$$
\eta_{A B}=\eta_{A}\left({ }^{3} P_{n}\right) \eta_{B}=\eta_{A} \eta_{(3 \bar{B})}\left({ }^{3} P_{l}\right)
$$

4. If $A \in \mathbb{H}^{m \times m}$ then there exists $A^{-1}$ if and only if there exist $\left(\phi_{A}\right)^{-1},\left(\mu_{A}\right)^{-1}$ and $\left(\eta_{A}\right)^{-1}$ and they are

$$
\phi_{A^{-1}}=\left({ }^{1} P_{m}\right) \phi_{A^{-1}}\left({ }^{1} P_{m}\right), \quad \mu_{A}{ }^{-1}=\left({ }^{2} P_{m}\right) \mu_{A^{-1}}\left({ }^{2} P_{m}\right)
$$

and

$$
\eta_{A}^{-1}=\left({ }^{3} P_{m}\right) \eta_{A^{-1}}\left({ }^{3} P_{m}\right)
$$

\subsection{Sylvester-s-Conjugate Matrix Equations over the Commuta- tive Quaternion Matrices}

For $A \in \mathbb{H}^{m \times m}, B \in \mathbb{H}^{n \times n}$ and $C \in \mathbb{H}^{m \times n}$, matrix equations $A\left({ }^{s} \bar{X}\right)-X B=$ $C,(s=1,2,3)$ are called Sylvester-s-Conjugate matrix equations on commutative quaternion matrices. In here, we construct some explicit expressions of the solutions of the matrix equations $A\left({ }^{s} \bar{X}\right)-X B=C$ by means of real representation of a commutative quaternion matrices and consimilarity of the two commutatative quaternion matrices. 


\section{i. Sylvester-1-Conjugate Matrix Equation $A\left({ }^{1} \bar{X}\right)-X B=C$}

In here, we investigate the solution of the Sylvester-1-conjugate matrix equation

$$
A\left({ }^{1} \bar{X}\right)-X B=C
$$

via the real representation, where $A \in \mathbb{H}^{m \times m}, B \in \mathbb{H}^{n \times n}$ and $C \in \mathbb{H}^{m \times n}$. We define the real representation of the matrix equation (17) by

$$
\phi_{A} Y-Y \phi_{B}=\phi_{C} .
$$

Theorem 13. The equation (17) has a solution $X$ if and only if the equation (18) has a solution $Y=\phi_{X}\left({ }^{1} P_{n}\right)$.

Theorem 14. The equation (18) has a solution $Y \in \mathbb{R}^{4 m \times 4 n}$ if and only if the equation (17) has a solution $X \in \mathbb{H}^{m \times n}$; in that case, if $Y \in \mathbb{R}^{4 m \times 4 n}$ is a solution to (18), then the matrix;

$$
X=\frac{1}{4}\left(\begin{array}{llll}
I_{m} & i I_{m} & j I_{m} & k I_{m}
\end{array}\right) Y^{\prime}\left(\begin{array}{c}
I_{n} \\
i I_{n} \\
j I_{n} \\
k I_{n}
\end{array}\right)
$$

is a solution to (17) where

$$
Y^{\prime}=\frac{1}{4}\left(Y\left({ }^{1} P_{n}\right)-Q_{m}^{-1} Y\left({ }^{1} P_{n}\right) Q_{n}+R_{m}^{-1} Y\left({ }^{1} P_{n}\right) R_{n}-S_{m}^{-1} Y\left({ }^{1} P_{n}\right) S_{n}\right) .
$$

Proof. We demonstrate that if the real matrix

$$
Y=\left(\begin{array}{cccc}
Y_{11} & Y_{12} & Y_{13} & Y_{14} \\
Y_{21} & Y_{22} & Y_{23} & Y_{24} \\
Y_{31} & Y_{32} & Y_{33} & Y_{34} \\
Y_{41} & Y_{42} & Y_{43} & Y_{44}
\end{array}\right), Y_{u v} \in \mathbb{R}^{m \times n}, u, v=1,2,3,4
$$

is solution to (18), then the matrix represented in (19) is a solution to (17). Since

$$
Q_{m}^{-1} \phi_{X} Q_{n}=-\phi_{X}, R_{m}^{-1} \phi_{X} R_{n}=\phi_{X}, S_{m}^{-1} \phi_{X} S_{n}=-\phi_{X} \text { and } Y=\phi_{X}\left({ }^{1} P_{n}\right)
$$

we have

$$
\begin{aligned}
& \phi_{A}\left(-Q_{m}^{-1} Y\left({ }^{1} P_{n}\right) Q_{n}\right)\left({ }^{1} P_{n}\right)-\left(-Q_{m}^{-1} Y\left({ }^{1} P_{n}\right) Q_{n}\right)\left({ }^{1} P_{n}\right) \phi_{B}=\phi_{C}, \\
& \phi_{A}\left(R_{m}^{-1} Y\left({ }^{1} P_{n}\right) R_{n}\right)\left({ }^{1} P_{n}\right)-\left(R_{m}^{-1} Y\left({ }^{1} P_{n}\right) R_{n}\right)\left({ }^{1} P_{n}\right) \phi_{B}=\phi_{C}, \\
& \phi_{A}\left(-S_{m}^{-1} Y\left({ }^{1} P_{n}\right) S_{n}\right)\left({ }^{1} P_{n}\right)-\left(-S_{m}^{-1} Y\left({ }^{1} P_{n}\right) S_{n}\right)\left({ }^{1} P_{n}\right) \phi_{B}=\phi_{C} .
\end{aligned}
$$


Last equations show that if $Y$ is a solution to (18), then

$$
\left(-Q_{m}^{-1} Y\left({ }^{1} P_{n}\right) Q_{n}\right)\left({ }^{1} P_{n}\right),\left(R_{m}^{-1} Y\left({ }^{1} P_{n}\right) R_{n}\right)\left({ }^{1} P_{n}\right)
$$

and

$$
\left(-S_{m}^{-1} Y\left({ }^{1} P_{n}\right) S_{n}\right)\left({ }^{1} P_{n}\right)
$$

are solutions to (18). Thus the undermentioned real matrix:

$$
Y^{\prime}=\frac{1}{4}\left(Y-\left(Q_{m}^{-1} Y\left({ }^{1} P_{n}\right) Q_{n}-R_{m}^{-1} Y\left({ }^{1} P_{n}\right) R_{n}-S_{m}^{-1} Y\left({ }^{1} P_{n}\right) S_{n}\right)\left({ }^{1} P_{n}\right)\right)
$$

is a solution to (18). If the right side of last equation multipled by $\left({ }^{1} P_{n}\right)$, then we get

$$
\phi_{X}=\left(\begin{array}{cccc}
Z_{0} & Z_{1} & Z_{2} & Z_{3} \\
Z_{1} & -Z_{0} & Z_{3} & -Z_{2} \\
Z_{2} & Z_{3} & Z_{0} & Z_{1} \\
Z_{3} & -Z_{2} & Z_{1} & -Z_{0}
\end{array}\right)
$$

where $\phi_{X}=Y\left({ }^{1} P_{n}\right)$ and

$$
\begin{aligned}
& Z_{0}=\frac{1}{4}\left(Y_{11}+Y_{22}+Y_{33}+Y_{44}\right), \quad Z_{1}=\frac{1}{4}\left(-Y_{12}+Y_{21}-Y_{34}+Y_{43}\right), \\
& Z_{2}=\frac{1}{4}\left(Y_{13}+Y_{24}+Y_{31}+Y_{42}\right), \quad Z_{3}=\frac{1}{4}\left(-Y_{14}+Y_{23}-Y_{32}+Y_{41}\right) .
\end{aligned}
$$

Thus, we get

$$
X=\frac{1}{4}\left(\begin{array}{llll}
I_{m} & i I_{m} & j I_{m} & k I_{m}
\end{array}\right) Y^{\prime}\left(\begin{array}{c}
I_{n} \\
i I_{n} \\
j I_{n} \\
k I_{n}
\end{array}\right)
$$

Theorem 15. Let $A\left({ }^{1} \bar{X}\right)-X B=0, X$ be regular and $A, B \in \mathbb{H}^{n \times n}$. Then $A$ is consimilar to $B$ according to 1 nd conjugate and $\phi_{A}$ is similar to $\phi_{B}$.

Proof. Since $X$ is reguler, we have $X^{-1} A\left({ }^{1} \bar{X}\right)=B$ from $A\left({ }^{1} \bar{X}\right)-X B=0$. Thus $A$ is consimilar to $B$ according to 1 nd conjugate. We can write from $X^{-1} A\left({ }^{1} \bar{X}\right)=B$

$$
\begin{gathered}
\phi_{\left(X^{-1}\right) A} \phi_{X}\left({ }^{1} P_{n}\right)=\phi_{B} \Rightarrow \phi_{\left(X^{-1}\right)}\left({ }^{1} P_{n}\right) \phi_{A} \phi_{X}\left({ }^{1} P_{n}\right)=\phi_{B} \\
\Rightarrow\left({ }^{1} P_{n}\right)\left(\phi_{X}\right){ }^{-1}\left({ }^{1} P_{n}\right)\left({ }^{1} P_{n}\right) \phi_{A} \phi_{X}\left({ }^{1} P_{n}\right)=\phi_{B}
\end{gathered}
$$


and

$\left({ }^{1} P_{n}\right)^{-1}\left(\phi_{X}\right)^{-1} \phi_{A}\left(\phi_{X}\left({ }^{1} P_{n}\right)\right)=\phi_{B} \Rightarrow\left(\phi_{X}\left({ }^{1} P_{n}\right)\right)^{-1} \phi_{A}\left(\phi_{X}\left({ }^{1} P_{n}\right)\right)=\phi_{B}$

Thus $\phi_{A}$ is similar to $\phi_{B}$.

ii. Sylvester-2-Conjugate Matrix Equation $A\left({ }^{2} \bar{X}\right)-X B=C$

Now, we investigate the solution of matrix equation

$$
A\left({ }^{2} \bar{X}\right)-X B=C
$$

by the method of real representation, where $A \in \mathbb{H}^{m \times m}, B \in \mathbb{H}^{n \times n}$ ve $C \in$ $\mathbb{H}^{m \times n}$. We first define the real representation matrix equation (23) by

$$
\mu_{A} Y-Y \mu_{B}=\mu_{C}
$$

In here $Y=\mu_{X}\left({ }^{2} P_{n}\right)$.

Theorem 16. The matrix equation (24) has a solution $Y \in \mathbb{R}^{4 m \times 4 n}$ if and only if the matrix equation (23) has a solution $X \in \mathbb{H}^{m \times n}$; In this case, if $Y$ is a solution to (24), then the matrix

$$
X=\frac{1}{4}\left(\begin{array}{llll}
I_{m} & i I_{m} & j I_{m} & k I_{m}
\end{array}\right) Y^{\prime}\left(\begin{array}{c}
I_{n} \\
-i I_{n} \\
-j I_{n} \\
k I_{n}
\end{array}\right)
$$

is a solution to (23) where

$$
Y^{\prime}=\frac{1}{4}\left(Y\left({ }^{2} P_{n}\right)+Q_{m}^{-1} Y\left({ }^{2} P_{n}\right) Q_{n}-R_{m}^{-1} Y\left({ }^{2} P_{n}\right) R_{n}-S_{m}^{-1} Y\left({ }^{2} P_{n}\right) S_{n}\right) .
$$

The proof of Theorem 16 may be proved along the same way as the proof of Theorem 14.

Theorem 17. Let $A\left({ }^{2} \bar{X}\right)-X B=0, X$ be regular and $A, B \in \mathbb{H}^{n \times n}$. Then $A$ is consimilar to $B$ according to 2 nd conjugate and $\phi_{A}$ is similar to $\phi_{B}$.

The proof of Theorem 17 may be proved along the same way as the proof of Theorem 15.

iii. Sylvester-3-Conjugate Matrix Equation $A\left({ }^{3} \bar{X}\right)-X B=C$

Lastly, we investigate the solution of matrix equation

$$
A\left({ }^{3} \bar{X}\right)-X B=C
$$

by the method of real representation, where $A \in \mathbb{H}^{m \times m}, B \in \mathbb{H}^{n \times n}$ ve $C \in$ $\mathbb{H}^{m \times n}$. We first define the real representation matrix equation (26) by

$$
\eta_{A} Y-Y \eta_{B}=\eta_{C}
$$

In here $Y=\eta_{X}\left({ }^{3} P_{n}\right)$. 
Theorem 18. The matrix equation (27) has a solution $Y \in \mathbb{R}^{4 m \times 4 n}$ if and only if the matrix equation (26) has a solution $X \in \mathbb{H}^{m \times n}$; In this case, if $Y$ is a solution to (27), then the matrix

$$
X=\frac{1}{4}\left(\begin{array}{llll}
I_{m} & i I_{m} & j I_{m} & k I_{m}
\end{array}\right) Y^{\prime}\left(\begin{array}{c}
I_{n} \\
i I_{n} \\
-j I_{n} \\
-k I_{n}
\end{array}\right)
$$

is a solution to (26) where

$$
Y^{\prime}=\frac{1}{4}\left(Y\left({ }^{3} P_{n}\right)-Q_{m}^{-1} Y\left({ }^{3} P_{n}\right) Q_{n}-R_{m}^{-1} Y\left({ }^{3} P_{n}\right) R_{n}+S_{m}^{-1} Y\left({ }^{3} P_{n}\right) S_{n}\right)
$$

The proof of Theorem 18 may be proved along the same way as the proof of Theorem 14 .

Theorem 19. Let $A\left({ }^{3} \bar{X}\right)-X B=0, X$ be regular and $A, B \in \mathbb{H}^{n \times n}$. Then $A$ is consimilar to $B$ according to 3 nd conjugate and $\phi_{A}$ is similar to $\phi_{B}$.

The proof of Theorem 19 may be proved along the same way as the proof of Theorem 15.

Example. Solve Sylvester-1-Conjugate matrix equation

$$
\left(\begin{array}{ll}
1 & i \\
i & j
\end{array}\right)\left({ }^{1} \bar{X}\right)-X\left(\begin{array}{ll}
1 & 0 \\
0 & 0
\end{array}\right)=\left(\begin{array}{cc}
-2 i+j & 2+j+k \\
1-i-k & 1+i
\end{array}\right)
$$

by using its real representation.

Real representation of given equation is

$$
\left(\begin{array}{cccccccc}
1 & 0 & 0 & 1 & 0 & 0 & 0 & 0 \\
0 & 0 & 1 & 0 & 0 & 1 & 0 & 0 \\
0 & 1 & -1 & 0 & 0 & 0 & 0 & 0 \\
1 & 0 & 0 & 0 & 0 & 0 & 0 & -1 \\
0 & 0 & 0 & 0 & 1 & 0 & 0 & 1 \\
0 & 1 & 0 & 0 & 0 & 0 & 1 & 0 \\
0 & 0 & 0 & 0 & 0 & 1 & -1 & 0 \\
0 & 0 & 0 & -1 & 1 & 0 & 0 & 0
\end{array}\right) Y-Y\left(\begin{array}{cccccccc}
1 & 0 & 0 & 0 & 0 & 0 & 0 & 0 \\
0 & 0 & 0 & 0 & 0 & 0 & 0 & 0 \\
0 & 0 & -1 & 0 & 0 & 0 & 0 & 0 \\
0 & 0 & 0 & 0 & 0 & 0 & 0 & 0 \\
0 & 0 & 0 & 0 & 1 & 0 & 0 & 0 \\
0 & 0 & 0 & 0 & 0 & 0 & 0 & 0 \\
0 & 0 & 0 & 0 & 0 & 0 & -1 & 0 \\
0 & 0 & 0 & 0 & 0 & 0 & 0 & 0
\end{array}\right)
$$




$$
=\left(\begin{array}{cccccccc}
0 & 2 & -2 & 0 & 1 & 1 & 0 & 1 \\
1 & 1 & -1 & 1 & 0 & 0 & -1 & 0 \\
-2 & 0 & 0 & -2 & 0 & 1 & -1 & -1 \\
-1 & 1 & -1 & -1 & -1 & 0 & 0 & 0 \\
1 & 1 & 0 & 1 & 0 & 2 & -2 & 0 \\
0 & 0 & -1 & 0 & 1 & 1 & -1 & 1 \\
0 & 1 & -1 & -1 & -2 & 0 & 0 & -2 \\
-1 & 0 & 0 & 0 & -1 & 1 & -1 & -1
\end{array}\right)
$$

If we solve this equation, we have

$$
Y=\left(\begin{array}{cccccccc}
0 & 1 & -1 & 0 & 0 & 1 & 0 & 0 \\
0 & 0 & 0 & -1 & 0 & 1 & -1 & 0 \\
1 & 0 & 0 & 1 & 0 & 0 & 0 & 1 \\
0 & 1 & 0 & 0 & 1 & 0 & 0 & 1 \\
0 & 1 & 0 & 0 & 0 & 1 & -1 & 0 \\
0 & 1 & -1 & 0 & 0 & 0 & 0 & -1 \\
0 & 0 & 0 & 1 & 1 & 0 & 0 & 1 \\
1 & 0 & 0 & 1 & 0 & 1 & 0 & 0
\end{array}\right)
$$

If we use the equation

$$
\phi_{X}=\frac{1}{4}\left(Y\left({ }^{1} P_{n}\right)-\left(Q_{m}^{-1} Y\left({ }^{1} P_{n}\right) Q_{n}-R_{m}^{-1} Y\left({ }^{1} P_{n}\right) R_{n}+S_{m}^{-1} Y\left({ }^{1} P_{n}\right) S_{n}\right)\right)
$$

We have

$$
\phi_{X}=\left(\begin{array}{cccccccc}
0 & 1 & 1 & 0 & 0 & 1 & 0 & 0 \\
0 & 0 & 0 & 1 & 0 & 1 & 1 & 0 \\
1 & 0 & 0 & -1 & 0 & 0 & 0 & -1 \\
0 & 1 & 0 & 0 & 1 & 0 & 0 & -1 \\
0 & 1 & 0 & 0 & 0 & 1 & 1 & 0 \\
0 & 1 & 1 & 0 & 0 & 0 & 0 & 1 \\
0 & 0 & 0 & -1 & 1 & 0 & 0 & -1 \\
1 & 0 & 0 & -1 & 0 & 1 & 0 & 0
\end{array}\right)
$$

Thus 


$$
\begin{aligned}
X & =\frac{1}{4}\left(\begin{array}{llllllll}
1 & 0 & i & 0 & j & 0 & k & 0 \\
0 & 1 & 0 & i & 0 & j & 0 & k
\end{array}\right)\left(\begin{array}{cccccccc}
0 & 1 & 1 & 0 & 0 & 1 & 0 & 0 \\
0 & 0 & 0 & 1 & 0 & 1 & 1 & 0 \\
1 & 0 & 0 & -1 & 0 & 0 & 0 & -1 \\
0 & 1 & 0 & 0 & 1 & 0 & 0 & -1 \\
0 & 1 & 0 & 0 & 0 & 1 & 1 & 0 \\
0 & 1 & 1 & 0 & 0 & 0 & 0 & 1 \\
0 & 0 & 0 & -1 & 1 & 0 & 0 & -1 \\
1 & 0 & 0 & -1 & 0 & 1 & 0 & 0
\end{array}\right)\left(\begin{array}{cc}
1 & 0 \\
0 & 1 \\
i & 0 \\
0 & i \\
j & 0 \\
0 & j \\
k & 0 \\
0 & k
\end{array}\right) \\
& =\left(\begin{array}{ll}
i & 1+j \\
k & i+j
\end{array}\right) .
\end{aligned}
$$

\section{References}

[1] W. R. Hamilton, Lectures on quaternions, Hodges and Smith, Dublin: 1853 .

[2] Y. TIAN, Universal factorization equalities for quaternion matrices and their applications, Math. J. Okoyama Univ. 41 (1999), 45-62.

[3] Y. TIAn, Similarity and consimilarity of elements in the real cayleydickson algebras, Adv. Appl. Clifford Algebras 9 (1999), 61-76.

[4] Y. TIAn, Solving two pairs of quadratic equations in quaternions, Adv. Appl. Clifford Algebras 20 (2010), 185-193.

[5] A. BAKER, Right eigenvalues for quaternionic matrices: a topological approach, Linear Algebra Appl. 286 (1999), 303-309.

[6] L. Huang And W. So, On left eigenvalues of a quaternionic matrix, Linear Algebra Appl. 323 (2001), 105-116.

[7] L. Huang, Consimilarity of quaternion matrices and complex matrices, Linear Algebra Appl. 331 (2001), 21-30.

[8] T. S. JIANG AND M. S. WEI, On a solution of the quaternion matrix equation $X-A \widetilde{X} B=C$ and its application, Acta Math. Sin. 21 (2005), 483-490. 
[9] T. S. JIANG AND S. LING, On a solution of the quaternion matrix equation $A \widetilde{X}-X B=C$ and its applications, Adv. Appl. Clifford Algebras 23 (2013), 689-699.

[10] C. SEGRE, The real representations of complex elements and extension to bicomplex, Systems. Math. Ann. 40 (1892), 40: 413.

[11] F. Catoni, R. Cannata and P. Zampetti, An introduction to commutative quaternions, Adv. Appl. Clifford Algebras 16 (2006), 1-28.

[12] S. C. Pei, J. H. Chang And J. J. Ding, Commutative reduced biquaternions and their fourier transform for signal and image processing applications, IEEE Transactions on Signal Processing 52 (2004), 20122031.

[13] S. C. Pei, J. H. Chang, J. J. Ding and M. Y. Chen, Eigenvalues and singular value decompositions of reduced biquaternion matrices, IEEE Trans. Circ. Syst. I 55 (2008), 2673-2685.

[14] T. Isokawa, H. Nishimura And N. Matsui, Commutative quaternion and multistate hopfield neural networks, In Proc. Int. Joint Conf. Neural Netw. (2010), 1281-1286.

[15] L. Guo, M. Zhu And X. Ge, Reduced biquaternion canonical transform, convolution and correlation, Signal Process 91 (2011), 2147-2153.

[16] H. H. Kosal and M. Tosun, Commutative quaternion matrices, Adv. Appl. Clifford Algebra 24 (2014), 769-779.

[17] H. H. Kosal, M. Akyigit and M. Tosun, Consimilarity of commutative quaternion matrices, Miskolc Math. Notes 16 (2015), 965-977.

Hidayet Huda Kosal

Department of Mathematics,

Faculty of Arts and Sciences, Sakarya University,

54187 Sakarya, Turkey,

E-mail: hhkosal@sakarya.edu.tr

Murat Tosun

Department of Mathematics,

Faculty of Arts and Sciences, Sakarya University,

54187 Sakarya, Turkey,

E-mail: tosun@sakarya.edu.tr 